\title{
Strates
}

STRATES Matériaux pour la recherche en sciences sociales

8 | 1995

La question de l'environnement: recherches parallèles en Espagne et en France

\section{Territorialiser la politique de l'environnement en}

France

Quels outils pour l'évaluation et l'aide à la décision?

\section{Aline Brochot}

\section{OpenEdition \\ Journals}

\section{Édition électronique}

URL : http://journals.openedition.org/strates/951

DOI : $10.4000 /$ strates.951

ISSN : $1777-5442$

\section{Éditeur}

Laboratoire Ladyss

Édition imprimée

Date de publication : 30 juin 1995

ISSN : 0768-8067

\section{Référence électronique}

Aline Brochot, «Territorialiser la politique de l'environnement en France », Strates [En ligne], 8 | 1995, mis en ligne le 20 décembre 2005, consulté le 08 septembre 2020. URL : http:// journals.openedition.org/strates/951; DOI : https://doi.org/10.4000/strates.951

Ce document a été généré automatiquement le 8 septembre 2020

Tous droits réservés 


\title{
Territorialiser la politique de l'environnement en France
}

\author{
Quels outils pour l'évaluation et l'aide à la décision?
}

\section{Aline Brochot}

1 La politique de l'environnement s'est structurée en France à partir de 1971, date de la création $\mathrm{du}$ premier ministère spécifique ${ }^{1}$. Conçue davantage comme une administration de mission, sorte de laboratoire d'idées et d'expertises, c'est une petite structure dans le dispositif gouvernemental français ${ }^{2}$ dont le rôle et les domaines d'intervention n'ont cependant cessé de s'élargir. Trois grands domaines lui sont désormais dévolus : la gestion des ressources naturelles, la protection de la nature et la prévention des pollutions et des risques ${ }^{3}$.

D'abord essentiellement centré sur la protection de milieux naturels spécifiques qui s'exerçait principalement sur des espaces délaissés par les autres activités, le champ d'action de l'environnement, tel qu'il s'est constitué au fil des années par ajouts et redéfinitions successives, est aujourd'hui très large et couvre des domaines divers qui vont de l'écologie scientifique, héritière des sciences naturalistes, à toutes les formes d'utilisation du sol et activités humaines dont le milieu naturel est le support, englobant tout ce qui a trait à la santé, au cadre de vie et au paysage ${ }^{4}$. Aussi la nécessité de considérer l'ensemble des facteurs de l'environnement dans une perception globale $\mathrm{du}$ territoire national est-elle désormais fortement ressentie.

Le ministère de l'Environnement a donc eu, au cours de ces vingt-trois années d'existence, à intégrer dans l'exercice de sa mission un nombre croissant de paramètres et par conséquent à mettre en oeuvre un nombre accru d'instruments de connaissance et d'observation. Il dispose aujourd'hui d'un système de connaissance complexe et diversifié qui couvre la majorité de ses domaines d'action ${ }^{5}$. Pourtant cette information reste sectorielle et partielle; une vision nationale qui permettrait d'apprécier, pour la totalité des espaces français, l'ensemble des phénomènes d'environnement dans leur ampleur et leur répartition spatiale fait encore défaut. En outre, une forte demande se dégage au sein de l'administration centrale ${ }^{6}$ pour la construction d'outils de connaissance et d'évaluation globale de la qualité «environnementale» des espaces, 
outils indispensables pour la formulation, l'orientation et la territorialisation ${ }^{7}$ d'une $^{\prime}$ politique nationale cohérente et, en fin de compte, pour l'exercice d'un rôle d'expertise essentiel dans le cadre national mais aussi international.

Nous essaierons donc, après l'analyse du système de connaissance actuel, d'examiner les conditions dans lesquelles un outil d'évaluation globale et nationale des espaces du point de vue de l'environnement pourra être construit et quelles pourraient en être les utilisations, au-delà de l'objectif premier de territorialisation des politiques sectorielles menées par le ministère de l'Environnement.

Quels outils de connaissance et d'observation?

La création d'un ministère de l'Environnement a fait naître la volonté de disposer d'un système d'information qui lui soit propre en mobilisant et en organisant les données relevant de son champ d'action. L'élaboration du système de connaissance actuel s'est opérée graduellement, par reprise d'éléments existants au sein d'autres institutions, par ajouts de nouveaux éléments grâce à la prise en compte de la dimension « environnement » dans les systèmes statistiques nationaux, et enfin par la création de données, propres ou sous-produits de l'activité administrative du ministère et des études scientifiques.

6 Inventaires, réseaux d'observation et de surveillance, séries statistiques, états cartographiques constituent les principaux types d'outils de connaissance et d'appréciation des situations à partir desquels la politique actuelle de l'environnement a été définie.

Inventaires

7 La pratique de l'inventaire, prise au sens large de dénombrement, n'est ni récente ni spécifique de l'environnement. De nombreux recensements nationaux d'éléments entrant dans le champ de l'environnement avaient été engagés bien avant l'émergence de préoccupations environnementales en France. Forêts, productions agricoles, cheptel, espèces animales et végétales, sites naturels sont recensés depuis parfois fort longtemps en fonction de leur importance stratégique, économique, culturelle ou scientifique. Dans bien des cas, il s'agit d'évaluer la ressource nationale que ces éléments représentent et de s'en assurer la maîtrise par une gestion appropriée. Ces inventaires, recensements ou enquêtes font partie, au moins partiellement, du système d'information de la politique de l'environnement soit en tant que données de cadrage de l'occupation du sol et des activités humaines ${ }^{8}$, soit parce que le domaine couvert fait partie du domaine de compétences de l'actuel ministère.

Avec le slogan " connaître pour gérer » le ministère de l'Environnement a lancé une politique d'inventaires nationaux spécifiques destinés à dresser en quelque sorte un « étatzéro » qui, plus qu'un simple état des lieux, doit permettre une appréciation qualitative de la réalité du patrimoine naturel national ${ }^{9}$. Le pas décisif dans cette démarche de connaissance exhaustive des milieux naturels français sera fait avec la loi de 1976 sur la protection de la nature. Elle stipule que «la protection des espaces naturels et des paysages, la préservation des espèces animales et végétales, le maintien des équilibres biologiques auxquels ils participent et la protection des ressources naturelles contre toutes les causes de dégradation qui les menacent sont d'intérêt général ». Dès lors, un vaste champ d'enquête et d'inventaire est ouvert puisque cette loi, en prévoyant des protections particulières de certains espaces et en déclarant d'intérêt général leur préservation, induit l'exigence d'une connaissance précise à la fois du patrimoine naturel national et des risques d'atteinte à son intégrité. 
9 Ainsi seront menés de front inventaire des milieux naturels considérés en tant que ressources naturelles et inventaire des éléments présentant des risques de dégradation pour celles-ci (voir tableau 1).

10 L'idée de réaliser un inventaire du patrimoine naturel français est lancée dès $1978^{10}$. Il s'agit avant tout de repérer les zones les plus remarquables du point de vue écologique pour les insérer dans les grands programmes de protection nationaux mais aussi internationaux ${ }^{11}$, européens principalement. Le ministre en confiera la réalisation au Secrétariat de la Faune et de la Flore ${ }^{12}$ qui, s'appuyant sur un réseau de naturalistes régionaux, livrera les premiers résultats nationaux en 1990 consacrant ainsi les ZNIEFF : Zones Naturelles d'Intérêt Écologique Faunistique et Floristique (voir encart).

11 Conçu comme un outil de référence, cet inventaire sert aujourd'hui de base à la définition de zones d'application de politiques, en particulier dans le cadre de directives européennes ${ }^{13}$ et, bien qu'il n'ait aucune valeur juridique (non opposabilité au tiers), il est largement consulté par les acteurs locaux - collectivités territoriales, associations -, en particulier dans le cadre des opérations d'aménagement (élaboration de documents d'urbanisme, schémas directeurs, études d'impact...).

12 Dans le domaine des risques et pollutions l'inventaire répond à deux objectifs. Il s'agit d'une part de dresser une liste de contrôle des éléments potentiellement dangereux et assurer ainsi la gestion du risque, et d'autre part de repérer les "points noirs » et établir des priorités d'action pour revenir à des seuils acceptables à la fois par les instances internationales et par une demande sociale toujours plus exigeante.

13 Les inventaires sont des éléments essentiels de l'aide à la décision politique. Ils permettent, par la vision nationale qu'ils apportent d'un phénomène, dans son ampleur et sa répartition, de dresser des états des lieux sous forme de listes et cartes, d'établir des hiérarchies régionales et de donner des priorités d'action. Ils permettent de repérer les extrêmes, d'établir des situations moyennes et des seuils critiques (les inventaires d'espèces, par exemple, ont permis d'établir des "listes rouges » d'espèces menacées ou en voie de disparition). Ils sont les instruments privilégiés pour le zonage des politiques sectorielles.

14 Cependant ils ne peuvent à eux seuls rendre compte de la globalité des situations et servir de base à l'établissement d'un diagnostic territorial. Leur opérationalité au niveau national se heurte en effet à quelques « effets pervers » :

15 "Effet de cloisonnement": ils restent sectoriels et sont rarement mis en relation avec d'autres éléments, les problèmes d'échelles de saisie de l'information étant souvent incontournables et rendant les comparaisons difficiles. Chaque recensement est pensé dans sa propre logique, sans tentative d'intégration dans un espace ou un système plus large par l'homogénéisation des unités d'observation et des échelles.

16 «Effet de nivellement»: ils ne permettent pas le plus souvent d'évaluation qualitative et de hiérarchisation entre les éléments recensés qui sont tous mis sur le même plan. Un exemple significatif est celui des sites classés auxquels le recensement donne le même poids quelle que soit leur valeur intrinsèque, qu'ils soient des sites prestigieux de valeur internationale ou des sites d'importance régionale ou locale. Le cas des ZNIEFF est un peu particulier dans le sens où les zones désignées sont déjà le produit d'une sélection et constituent donc une évaluation en soi.

17 «Effet arrêt sur image » : enfin, leur délai de mise en oeuvre et de réalisation et les coûts entraînés par l'ampleur de telles opérations lorsqu'elles sont pratiquées au niveau 
national ne permettent pas le plus souvent d'actualisation régulière, empêchant ainsi toute vision dynamique du phénomène observé et figeant une représentation à un temps T. L'Inventaire Permanent du Littoral illustre parfaitement ce propos, les observations et relevés ayant cessé depuis 1982, principalement faute d'accord sur les participations financières des régions concernées.

Réseaux d'observation et de surveillance

«Observer pour prévenir », tel pourrait être le slogan général ici. Il ne s'agit plus seulement de localiser et de recenser mais d'observer et surveiller en temps continu et tenter de prévoir des évolutions dans un but de prévention, d'assurer la gestion du risque, de passer de la veille à l'alerte, les phénomènes observés relevant essentiellement des risques industriels et technologiques et des pollutions.

La qualité des sols, des eaux superficielles et marines, de l'air, l'état sanitaire des forêts sont appréhendés par la mise en place de réseaux de points d'observation et de mesure localisés en des lieux représentatifs ou stratégiques (voir tableau 2). Les réseaux de surveillance ont été progressivement mis en place au fur et à mesure de la mise en évidence de dysfonctionnements et de la réglementation édictée pour y remédier. Ainsi, dès 1964, la loi relative "au régime, à la répartition et à la lutte contre la pollution des eaux» prévoit la réalisation d'un système de mesure (qualifié improprement d'inventaire) de la qualité des eaux superficielles. Le premier « inventaire de la qualité des eaux superficielles » sera réalisé en $1971^{14}$ et est repris depuis dans le cadre du Réseau National de Bassin.

Plus adaptés à des phénomènes à développement rapide, à temps de réponse brefs, par la régularité de leur système de mesure, les réseaux de surveillance mis en place répondent à l'exigence de contrôle et de suivi régulier nécessaires dans le cadre de la gestion des risques technologiques ou industriels mais ils souffrent malgré tout de certaines faiblesses.

21 Du fait de la relative "jeunesse " du système d'observation et d'analyse, on reste malgré tout dans le domaine de l'expérimentation. En effet, on ne dispose encore que de peu de références dans le temps - le temps-zéro est celui de la mise en place du système -, ce qui interdit toute tentative de modélisation et pose de gros problèmes d'établissement de normes fiables et reconnues. L'introduction croissante des directives européennes ou des conventions internationales en matière de limitations des risques vient peu à peu combler ce manque en fixant les seuils et limites à respecter ${ }^{15}$.

22 Par ailleurs, certains phénomènes -comme la pollution diffuse des sols- ont des temps d'apparition et de développement difficilement saisissables, du moins à l'échelle de vie humaine. Ce qui impose la recherche de méthodes de "dépistage précoce» des phénomènes d'altération de manière à pouvoir intervenir en temps utiles sur les causes, ou bien pour apporter des mesures correctives appropriées.

Les réseaux de surveillance tendent également à favoriser l'existence de ce que l'on peut appeler des "zones d'ombre », dans le temps, dans l'espace et dans les paramètres suivis.

Paradoxalement, malgré leur régularité et leurs objectifs mêmes d'observation en continu, la fréquence des mesures se révèle parfois insuffisante pour rendre compte de situations souvent très variables dans le temps. C'est en particulier le cas pour les conditions hydrologiques - eaux superficielles et marines-, sensibles au moindre bouleversement (pollutions ponctuelles et soudaines, orages, etc.) 

des phénomènes à temps d'apparition rapide, mais un tel système atteint vite ses limites lorsqu'il s'agit de passer de la veille à l'alerte en cas d'accidents (pollution accidentelle, crues rapides...). S'ils offrent l'avantage de structures légères, parfois entièrement automatisées et qui peuvent s'appuyer sur un petit nombre de personnes, cela pose le problème du seuil critique de présences et de relais nécessaires pour assurer la liaison entre la surveillance, le repérage du franchissement de la cote d'alerte, la décision de diffusion de l'alerte et sa transmission aux populations des zones exposées. Les inondations de l'hiver 1993/94 et de l'automne 1994 en France où certaines populations ont été littéralement prises au piège en sont l'exemple. Peut-être faut-il voir dans ce cas précis la manifestation d'effets pervers, d'un côté du dispositif de surveillance qui favorise l'observation des grandes rivières et fleuves au détriment des petits cours d'eau et fait que, lorsque le seuil critique est atteint sur les fleuves, il est déjà trop tard pour leur amont, mais aussi d'un autre côté, de la décentralisation qui a multiplié les niveaux de responsabilité et rendu obligatoire un nombre peut-être «superflu» de relais administratifs avant que l'information du danger ne parvienne aux populations concernées.

\section{Statistiques}

Dans l'espace, le faible nombre de points de prélèvement constitue le principal obstacle à la représentativité des mesures. Ces observations ne peuvent avoir valeur de sondage et en aucun cas on ne peut extrapoler à partir d'un point d'observation pour d'autres s'effectuer que dans le temps et point par point. En outre, si elles renseignent sur les fluctuations de la qualité observée pour chaque point, ces mesures ne rendent pas ensuite de leur trajectoire. C'est ici que se situe par exemple tout le problème de la mesure de la pollution des nappes phréatiques par les nitrates d'origine agricole.

rendre compte de la complexité des interactions et ne permettent pas encore d'appréhender certaines formes de pollution déjà anciennes (certains métaux) ou mises à jour plus récemment (phosphates, nitrates, pesticides...)

certaine "culture de l'extrême». La surveillance se fait le plus souvent sur les "points noirs »: littoraux à risques (estuaire de la Seine, Étang de Berre...), zones de fortes agglomérations etc., au prix d'investissements parfois très lourds, mais on sait en fin de compte peu de choses sur des espaces plus «ordinaires »-c'est-à-dire la grande par exemple que l'état général des petits cours d'eau tend à se dégrader, mais qu'ils ne sont pas ou peu surveillés ${ }^{16}$.

La création d'un ministère propre n'a pas contribué à la construction d'un outil statistique spécifique. En 1972, un groupe interministériel d'évaluation de l'environnement fut mis en place afin d'examiner les conditions dans lesquelles un système complet de données sur l'environnement pouvait être construit. L'orientation principale de ses conclusions fut alors de ne pas créer un nouveau système d'enquêtes statistiques spécifiques de l'environnement, mais de faire apparaître une dimension nouvelle dans les systèmes déjà existants ${ }^{17}$. Les principales données sur 
l'environnement sont donc restées réparties entre plusieurs ministères: Agriculture, Équipement, Tourisme, Santé, Culture... (voir tableau 3).

Dès lors deux types de données peuvent aujourd'hui être distinguées dans le dispositif statistique de l'environnement ${ }^{18}$ :

Des données venues d'ailleurs: Rassemblées et exploitées par d'autres départements ministériels par lesquels et pour l'exercice desquels elles ont été conçues, ce sont davantage des données de cadrage, "l'environnement de l'environnement», qui servent de support, d'argumentation à l'analyse. Le champ couvert déborde largement la seule protection de la nature et des écosystèmes, pour prendre en compte l'ensemble des interactions entre activités humaines et milieu naturel : urbanisation, agriculture, activités productives, transport, tourisme, santé publique...

2 Des statistiques, sous-produits de la gestion administrative: Les principales séries statistiques effectivement produites par le ministère ne sont en fait souvent que des sous-produits de l'action administrative de ses services et correspondent aux bilans des actions engagées (procédures réglementaires, acquisitions, procédures contractuelles...) dans ses différents domaines d'intervention : protection de la nature, gestion de ressources naturelles, prévention des risques et pollution.

33 L'intérêt des statistiques réside dans leur aptitude à traduire des dynamiques, à repérer des tendances, facultés qui prennent toute leur importance dans un domaine où l'objectif fondamental est la prévention. Elles permettent par ailleurs, lorsqu'elles sont régionalisées, de mettre en comparaison des territoires, d'établir des catégories ou des hiérarchies d'espaces. Aussi la difficulté d'élaborer un véritable système statistique sur l'environnement tient-il à deux problèmes majeurs de ce champ de recherche : celui de sa relative «jeunesse » et celui des échelles d'appréhension des phénomènes.

4 L'environnement, considéré comme un champ d'études et d'action politique à part entière, en est encore à sa prime jeunesse et toujours en cours de structuration ${ }^{19}$ et l'analyse des évolutions souffre de l'extrême faiblesse des séries rétrospectives qui empêche la formulation de conclusions solides sur la nature et les effets des interactions entre systèmes naturels et systèmes sociaux. Toute tentative prospective repose alors essentiellement sur des avis d'experts.

5 Les phénomènes d'environnement se développent sur des pas de temps très variables (un accident de pollution se développe en quelques minutes, un champ laissé à l'abandon se reboisera sur plusieurs dizaines d'années, etc.) rendant l'homogénéisation du choix des périodicités d'observation et de saisie des données délicate. Chaque série est alors établie selon le pas de temps le plus approprié au phénomène considéré. Pas plus d'homogénéité n'est possible dans le choix des unités spatiales : du parcellaire au planétaire, du point localisé à la zone, du biotope à la biosphère sont autant de gradients le long desquels peuvent se décrire les phénomènes d'environnement. Il est difficile dans ces conditions de résoudre le problème de la définition des zones géographiques adaptées à la mesure de l'environnement. Chaque élément est donc saisi selon l'échelle qui lui est la plus appropriée.

Devant ces difficultés, on ne peut que se reporter sur des échelles, certes arbitraires mais déjà éprouvées pour l'observation d'autres phénomènes, et qui permettent l'harmonisation de la collecte et de la saisie des données: mois, années, unités administratives, etc. demeurent les cadres privilégiés de la saisie des données, même si, 
à l'aval, émergent d'autres problèmes: superpositions, emboîtements, chevauchements...

États cartographiques

37 La pratique de la représentation cartographique, pour ancienne qu'elle soit, représente encore aujourd'hui un des outils essentiels de la connaissance et de l'aide à la décision en matière d'analyse de l'espace. La politique de l'environnement y a largement recours soit comme outil de référence, soit comme outil de travail préalable au choix, soit comme outil de gestion.

La carte est un outil de référence essentiel pour la représentation des éléments de l'environnement dont beaucoup sont liés au milieu physique, donc plus statiques. Elle est en outre parfois la seule méthode efficace, pour la représentation de certains éléments difficilement saisissables sous une autre forme comme, par exemple, tous les linéaires (réseau hydrographique, routier, haies et linéaires boisés...). La vision immédiate qu'elle offre de la répartition, de l'étendue d'un phénomène en fait un objet de consultation absolument essentiel pour toute opération où la prise en compte de l'environnement est requise. Cartes des zones inondables, des zones d'avalanches, des zones à risque sismique, des ZNIEFF, etc. sont ainsi des documents de base et de cadrage indispensables.

Par la superposition d'éléments de nature différente sur un même espace, la carte permet l'analyse de phénomènes interdépendants et une appréciation plus globale de l'état ou de la destination d'un territoire. État des lieux ou support de scénario, elle est un outil de travail préalable au choix qui fait partie intégrante de l'éventail d'instruments utilisés dans la formulation et l'affichage des choix et priorités des politiques de l'environnement. Citons les Plans d'Exposition aux Risques, Schémas d'Aménagement et de Gestion de l'Eau, etc. Par la mise en comparaison des situations régionales elle permet la définition des politiques nationales, comme par exemple le choix des zones à protéger.

Nombreuses sont désormais les réglementations qui s'appuient sur un état cartographique. Outil de gestion, la carte devient un document contractuel lorsqu'une opération repose sur la délimitation d'un territoire sur lequel des dispositions particulières vont s'appliquer. L'établissement et l'approbation par l'État de la Charte d'un Parc naturel régional, par exemple, repose sur la délimitation préalable d'un territoire dans lequel ses principes devront être observés. La carte (ou le plan) peut même devenir un objet juridique à part entière qui peut s'imposer en cas de conflit entre deux parties. C'est le cas des POS (Plans d'Occupation des Sols) ou de toutes les délimitations d'espaces protégés qui sont généralement annexées au décret de protection (réserves naturelles, sites classés, parcs nationaux...).

41 On le voit, ce rapide inventaire des utilités et utilisations de la cartographie justifie largement l'importance qui lui est donnée dans tous les compartiments de la politique de l'environnement et en fait un outil complet, même s'il comporte certaines limites liées :

42 - à la nature des paramètres cartographiés qui doivent être objectifs et stables. Comment traduire les éléments subjectifs ou comportant trop de variabilité ?

43 - au nombre limité d'éléments représentés pour que la carte reste lisible ;

44 - à une certaine impression de « figé » en l'absence d'actualisation périodique.

Pour une vision globale et nationale de la qualité de l'environnement 

patrimoine naturel et culturel riche» qu'il importe de protéger par une gestion adaptée. Si l'élaboration du projet de Parc naturel se fait au niveau régional, l'agrément final est donné par le ministre de l'Environnement sous forme d'un label accordé pour une période de dix ans. Le ministre doit donc être en mesure d'opérer un arbitrage entre les différents projets qui lui sont présentés ${ }^{22}$ en s'assurant de la conformité, non seulement des objectifs des Chartes qui devront les régir, mais également des caractéristiques des territoires désignés. Il doit dès lors pouvoir disposer d'une base d'évaluation nationale pour confronter, hiérarchiser, établir des priorités et tenter de planifier une répartition géographique harmonieuse de «l'Institution-parc». Car plusieurs questions se posent aujourd'hui pour l'avenir de cette politique. Jusqu'à combien de Parcs naturels est-il raisonnable d'aller? Doit-on fixer un objectif quantitatif, un chiffre-clé2 ${ }^{23}$ ? Doit-on se diriger vers une représentation régionale, voire départementale ou bien vers un échantillonnage représentatif des milieux naturels, types paysagers et espaces culturels français?

51 Le besoin d'un outil d'évaluation nationale des territoires est donc clairement défini dans le cadre d'une politique sectorielle, mais il s'affirme également de plus en plus par l'introduction des règlements européens qui nécessitent la réalisation de zonages et la désignation, de la part des États-membres, de zones éligibles aux divers programmes communautaires.

Vers un système d'indicateurs de l'environnement et du paysage 

de l'Environnement (IFEN) ${ }^{24}$, pour parvenir à la construction d'un outil d'évaluation nationale performant s'orientent vers l'élaboration d'une critériologie spécifique qui, grâce à la mobilisation d'indicateurs pertinents au sein d'une base de données unique, à leur suivi et à leur traitement statistique, permettrait, d'une part de représenter les différents aspects de l'environnement pris dans sa plus grande acception, c'est-à-dire en y incluant les dimensions paysagère et patrimoniale et, d'autre part d'apprécier contradictoirement les situations régionales sur tout le continuum territorial. d'un ensemble de données qui, nous l'avons vu, sont de natures diverses, mobilisées selon des logiques d'utilisation différentes et éparpillées entre des gestionnaires multiples. Après une première phase d'inventaire des données disponibles, d'identification des partenaires concernés et de réflexion sur la faisabilité d'un tel projet, trois choix fondamentaux restent à fixer: le choix des échelles, le choix des indicateurs à retenir et le choix des méthodes d'exploitation les plus adaptées aux objectifs poursuivis.

Les échelles de mobilisation des données

54 L'échelle spatiale doit pouvoir être fixée à un niveau territorial de base qui soit significatif et suffisamment fin pour pouvoir, par agrégation, en former d'autres plus étendus et ainsi rendre compte du continuum dans lequel s'inscrivent les phénomènes d'environnement. Trois catégories d'unités spatiales sont couramment employées dans l'appréhension et l'observation de ces phénomènes : entité administrative (commune, canton, etc. ); découpage géométrique de l'espace (par carroyage); et entité «naturelle », c'est-à-dire une zone délimitée selon des critères physiques, telle que le bassin-versant.

Le choix final ne sera pas indépendant de l'utilisation finale de cette critériologie (description à visée scientifique ou aide à la décision politique), ainsi que du niveau géographique auquel les principales données de l'environnement sont actuellement saisies. Car il sera sans doute opportun, dans un but d'évaluation globale, d'y insérer des paramètres issus d'autres champs de connaissance (socio-économie, culture...) pour les mettre en relation ou mettre en évidence leurs interactions. Il sera alors nécessaire de disposer d'une échelle commune. De ce point de vue, les unités administratives semblent les plus indiquées car elles présentent l'avantage d'offrir un dénominateur commun entre la majorité des bases de données existantes et permettent le rassemblement des indicateurs le long d'un gradient qui couvre tout le territoire (de la commune au canton, au département, et à la région).

Pour les échelles de temps, la difficulté réside essentiellement dans la réduction à une même échelle temporelle de phénomènes de nature différente qui se développent et s'apprécient sur des pas de temps extrêmement variables (reboisement ou accident de pollution). Peut-être faut-il envisager la constitution de groupes de critères mesurables à des pas de temps différents selon leur nature (tous les ans, 5 ans...) ?

Les indicateurs représentatifs

57 Les spécificités des problèmes d'environnement et leur large spectre font que l'évaluation doit pouvoir prendre en compte aussi bien le statique que le dynamique, l'amont, l'aval et la périphérie, l'objectif et le sensible, le local et le global... et leurs articulations. Il s'agit alors de sélectionner des indicateurs qui soient représentatifs et rendent compte de la qualité intrinsèque et de la dynamique des territoires et des 
éléments de risques pour leur intégrité. Cela suppose que soient identifiées toutes les composantes qui participent à «l'état de l'environnement». Les principaux éléments sont connus grâce aux inventaires, réseaux, bases statistiques déjà en place, qui représentent le «stock » dans lequel les données pourront être puisées.

On distingue traditionnellement deux types d'indicateurs: des indicateurs d'état dont la fonction est de caractériser aussi bien quantitativement que qualitativement, et des indicateurs de tendance aptes à rendre compte des dynamiques et de leurs composantes.

Le choix final dépendra à la fois de la possibilité de résoudre les problèmes de mobilisation des données à l'échelle géographique souhaitée et de la pertinence de chaque indicateur à cette échelle pour l'évaluation et la hiérarchisation.

La méthodologie d'exploitation

60 Les objectifs assignés à l'exploitation de cette base de données sont clairs. Elle devra permettre :

61 - d'analyser comparativement dans le temps et dans l'espace les situations régionales;

62 - de situer les risques, d'évaluer les potentialités, et d'établir des priorités et stratégies d'action ;

63 - d'évaluer l'efficacité des politiques menées et de justifier les choix territoriaux faits pour leur application ;

64 - de mettre en perspective des situations par l'élaboration de scénarios tendanciels.

65 Cette problématique a déjà été soulevée dans de nombreux domaines, en particulier ceux ayant trait à l'aménagement du territoire et au développement régional, et diverses méthodes d'évaluation et de diagnostic territorial y ont été développées, soit dans un objectif de caractérisation et qualification des espaces, soit dans un but d'identification et de désignation de territoires répondant à certains critères ou présentant des caractéristiques communes. Nous les décrirons brièvement, chacune mettant en oeuvre des instruments et une chaîne d'opérations qui lui sont propres.

66 "Grille de lecture » : Il s'agit de qualifier, de porter un jugement sur un espace délimité au moyen d'une "grille de lecture » qui fournit un ensemble d'indicateurs d'état, d'évolution et de points de référence (moyennes nationales, régionales...), par rapport auxquels la situation de la zone étudiée va être évaluée ${ }^{25}$. C'est donc une évaluation relative qui permet le positionnement de l'espace considéré par rapport à ses voisins immédiats ou par rapport à des espaces de référence sur lesquels la même grille sera appliquée. Ce type de démarche est utilisé en particulier dans le cadre du développement régional en vue d'élaborer des plans d'actions et stratégies de mise en valeur d'un territoire.

67 Analyse multicritères: Par la mise en oeuvre et le traitement statistique d'un ensemble d'indicateurs représentatifs régionalisés, elle permet de faire apparaître des catégories d'espaces qui se "ressemblent", qui ont des caractéristiques communes et ainsi d'élaborer des typologies régionales. Cette méthode a déjà été largement explorée, en particulier dans le cadre de l'application des politiques de l'aménagement du territoire qui s'appuient souvent sur une délimitation préalable de zones possédant en commun un ensemble de caractéristiques: délimitation des zones "défavorisées ", des zones éligibles aux objectifs européens (5b) ${ }^{26}$.

68 Elle présente des avantages certains dans une optique de comparaison nationale en donnant une image globale, sorte de «vue d'avion» du territoire national qui permet 
d'élaborer une échelle de valeur nationale par l'appréciation de tous les cas de figure représentés, de mettre en comparaison de façon plus objective et plus neutre des territoires entre eux et par rapport à l'image nationale, favorisant ainsi le choix des priorités à accorder, et enfin de faire apparaître de nouveaux espaces présentant des caractéristiques homogènes qui pourront être indépendants des limites administratives.

Les domaines de l'évaluation paysagère et de l'analyse des représentations fournissent également des exemples de méthodes d'évaluation des territoires. Principalement à base d'appréciation visuelle in situ et d'enquêtes individuelles et s'appliquant sur des espaces généralement restreints, elles font, le plus souvent, largement appel à la subjectivité de la perception des caractéristiques et de la valeur d'un espace. L'introduction du sensible pose alors le problème de la généralisation de l'analyse au cadre national, et en particulier celui de l'élaboration d'une échelle de valeur qui soit reconnue par l'ensemble du corps social. Ces types d'analyses demeurent cependant de bons moyens d'appréhension d'une réalité quotidienne et immédiatement perceptible de la qualité d'un espace donné par l'analyse des jugements portés par ses différents acteurs.

L'utilisation de l'une ou l'autre de ces méthodes dépendra bien sûr largement à la fois de l'objectif de l'analyse et du niveau géographique de la recherche ou de la réflexion. Toutes ne sont pas opératoires à toutes les échelles et offrent des degrés de pertinence qui varient en fonction de l'espace observé. Une analyse statistique multicritères convient mieux à une évaluation s'appliquant sur un grand nombre d'unités territoriales et n'aurait que peu de sens au niveau local; l'utilisation d'une grille de lecture est plus appropriée à l'analyse régionale tandis que les analyses paysagères et des représentations restent des instruments d'évaluation locale voire micro-régionale.

71 C'est donc principalement de ces méthodes, déjà éprouvées dans d'autres cadres, que la politique de l'environnement pourra s'inspirer pour bâtir son propre outil d'évaluation. Elle pourra également s'appuyer sur le développement et la généralisation de technologies de pointe comme la télédétection et ses interprétations cartographiques ou l'enregistrement direct et en temps réel au sein de bases de données des informations transmises par des capteurs, qui constituent déjà des avancées intéressantes. Les développements futurs de CORINE Land Cover ${ }^{27}$ pourront sans doute apporter une solution à l'observation globale et en continu des espaces. Les développements récents de l'outil informatique permettent en outre d'envisager de sérieuses avancées dans la collecte, le traitement et la représentation graphique de données territorialisées avec, en particulier, la mise en place de Systèmes d'Information Géographique.

Quels objectifs généraux assigner à une critériologie de l'environnement ?

La disponibilité d'une critériologie spécifique constitue un enjeu majeur pour l'avenir et représente l'outil de base à partir duquel, par la mise en oeuvre de méthodes d'exploitation appropriées, le territoire national pourra être exploré, qualifié, évalué. Dès lors, au-delà de la simple application de politiques sectorielles, il est permis d'assigner à cette base de données des finalités plus larges et plus ambitieuses. Par son côté global, elle pourra servir des objectifs plus généraux de définition ou d'orientation de la politique nationale de l'environnement et ainsi faire prendre tout son sens au slogan « Penser globalement pour agir localement ». 
73 La mise en évidence des situations régionales quant à la qualité globale de leur environnement donnerait au ministère une base de discussion solide face à d'autres départements ministériels dont les logiques d'actions sont parfois à l'opposé de celles de préservation et de protection.

74 Par ailleurs, elle constituerait, vis-à-vis d'une demande sociale qui s'exprime principalement par l'exigence d'une information précise sur la qualité de l'environnement et la réalité des risques encourus, une base de concertation plus rationnelle entre particuliers, collectivités locales et pouvoirs publics.

75 Le monde scientifique est également largement intéressé par l'exploitation de cette critériologie qui pourrait ouvrir ou aider à développer de nouveaux axes de recherche, notamment en matière d'analyse de l'espace, par l'identification de types et catégories d'espaces caractéristiques par exemple..

76 Enfin, son exploitation sur plusieurs années apporterait sans doute des éclairages précieux sur les dynamiques en cours et instituerait un dispositif de «veille scientifque » qui permettrait d'identifier les processus à l'oeuvre en en repérant les causes et impacts.

Conclusion

77 La politique de protection de l'environnement a été le plus souvent pensée dans l'urgence, de façon pragmatique, ponctuelle, sectorisée et discontinue, quelquefois imposée par la force des événements, ou par la pression d'une demande sociale ellemême désorientée par l'afflux d'informations contradictoires où le raisonné cède souvent au catastrophisme.

78 Chaque segment de cette politique a donné lieu à une multiplication de directives, de dispositions juridiques qui se juxtaposent ou se superposent dans le temps et sur le territoire et répondent à des finalités spécifiques. Plusieurs systèmes d'information ou d'expertise ont été construits de manière parallèle : inventaires scientifiques, données techniques collectées pour la gestion, produits ou sous-produits statistiques ou comptables, représentations cartographiques, résultats d'études... sans que le ministère n'ait pu disposer d'un réel pouvoir de mise en cohérence globale de tous ces outils.

79 Parvenu dans sa phase de maturation, le ministère de l'Environnement doit explorer de nouvelles voies d'approche pour la connaissance et l'évaluation environnementale des espaces français en termes quantitatifs mais aussi qualitatifs. La volonté affichée de rassembler l'ensemble des données disponibles dans une base de données unique et de rechercher et tester de nouvelles méthodes d'évaluation et d'analyse est clairement manifestée par la création de l'IFEN dont ce sont les missions principales. Les moyens suivront-ils la décision politique ? Par ailleurs, la validité et l'opérationalité d'une telle démarche critériologique renvoie à quelques questions de fond spécifiques des problèmes d'évaluation de l'environnement. En effet, est-il pensable de renseigner chaque point ou maille du territoire sur toutes les composantes de l'environnement? Est-il possible de saisir et de restituer statistiquement la complexité des interactions à l'oeuvre en matière d'environnement? Est-il possible de traduire la qualité « environnementale » d'un espace en l'absence d'un système de normes et d'échelles de valeur formellement établi? Ou bien est-il envisageable de s'en tenir au suivi de quelques indicateurs synthétiques, sorte d'indicateurs de la «conjoncture environnementale » comme il existe un indicateur de la conjoncture économique, à partir de l'observation en continu d'un certain nombre de paramètres significatifs? 
Autant de questions qui ne trouveront leur réponse que dans l'expérimentation. La recherche scientifique est, quant à elle, largement sollicitée et impliquée, soit dans l'élaboration de bilans de l'action accomplie ${ }^{28}$, soit dans la recherche de nouvelles voies et méthodes d'analyse et d'évaluation ${ }^{29}$.

\section{BIBLIOGRAPHIE}

ADER Gérard (1982), Les Statistiques de l'environnement, Rapport au ministre de l'Environnement, Paris, INSEE.

AITCHISON John W., BONTRON Jean-Claude \& BROCHOT Aline (1983), Les Espaces-parcs : Analyse socio-économique des parcs naturels régionaux, Paris, SEGESA/Ministère de l'Environnement.

BARNIER Michel (1991), Le Défi écologique. Chacun pour tous, Paris, Hachette, coll. Pluriel. BONTRON Jean-Claude, BROCHOT Aline \& LUGINBÜHL Yves (1994), Les Indicateurs du paysage. Étude de cadrage, faisabilité d'une critériologie, Paris, STRATES/SEGESA - IFEN.

Les Comptes du patrimoine naturel, Collections de l'INSEE, série C, n 137-138, décembre 1986.

«L'Économie de l'environnement », Économie et Statistique, 258-259, oct/nov. 1992.

L'État de l'environnement. Édition 1991-1992, Paris, La Documentation française.

CHABASON Lucien (dir.), «L'environnement et les espaces ruraux », in L'Aménagement de l'espace rural, École nationale d'Administration, promotion Léon Gambetta (1991-1993), Paris, La Documentation Française, 2 volumes.

Guide du diagnostic pour le développement rural (1991), SEGESA/Commissariat Général du Plan. Histoire, évaluation et prospective des systèmes d'information statistique, Rapport du groupe de réflexion à long terme « Espace et environnement » du Conseil National de l'Information Statistique, septembre 1988.

JOLLIVET Marcel \& PAVE Alain (1993), «L'environnement, un champ de recherche en formation », Paris, Natures Sciences Sociétés, vol. 1, 1, pp. 6-20.

« La Qualité des eaux superficielles : Quelle évolution depuis vingt ans ? », IFEN, Les Données de l'environnement, 1, janv. 1994.

LARRUE Corinne \& KNOEPFEL Peter (1993), Évaluation de la politique française de surveillance et de connaissance de l'état de l'environnement, L'OEIL-IDHEAP/Ministère de l'Environnement-Centre de Prospective et de Veille scientifique.

MAURIN Hervé (1992), «Connaissance de la faune et de la flore sauvages : organisation, bilan et perspectives ", L'État de l'environnement, op. cit.

PRIEUR Michel (1991), Droit de l'environnement, Paris, Dalloz.

THEYS Jacques (1992), Environnement et aménagement du territoire dans les années 1990-2010:

l'exigence écologique, Paris, DATAR. 


\section{ANNEXES}

\section{L'inventaire des ZNIEFF}

Lancé en 1982 à l'initiative de la Direction de la Nature et des Paysages du ministère de l'Environnement, l'inventaire des ZNIEFF (Zones Naturelles d'Intérêt Écologique Faunistique et Floristique) s'est achevé dans sa première phase en 1990. Mis en oeuvre dans toutes les Régions par un important réseau de chercheurs naturalistes (4000 spécialistes environ), selon une méthode définie à l'échelon national et coordonné par des comités régionaux, il a pour but de localiser les zones présentant un intérêt particulier du point de vue écologique.

L'année 1994 a vu le lancement d'une deuxième phase d'inventaire qui doit permettre de valider les résultats du premier passage, et en particulier de corriger certaines différences régionales apparues dans l'appréciation et la désignation des zones, et d'affiner encore la connaissance des milieux naturels par la mise en place d'une nouvelle procédure de collecte des données s'appuyant sur une typologie revue et améliorée.

Deux types de zones sont identifiées :

- Les zones de Type I sont caractérisées par leur intérêt biologique remarquable (présence d'espèces protégées, associations d'espèces races, menacées ou caractéristiques du patrimoine régional).

- Les zones de Type II sont des grands ensembles naturels, riches et peu modifiés, qui offrent des potentialités biologiques importantes (elles peuvent inclure des zones des type I).

Au 30 septembre 1992, 14600 zones étaient décrites en France métropolitaine, enregistrées et traitées dans une base de données informatisée au sein du Secrétariat de la Faune et de la Flore. Près de $30 \%$ du territoire métropolitain est couvert par des ZNIEFF ( $20 \%$ de type II et $8,2 \%$ de type I).

\begin{tabular}{|l|l|l|}
\hline Type & Nombre & Surface $(\mathbf{k m} 2)$ \\
\hline \hline I & 12618 & 44.190 \\
\hline \hline II & 1982 & 108420 \\
\hline \hline I+II & 14600 & 152610 \\
\hline
\end{tabular}

Cette banque de données constitue pour les administrations et les élus un outil d'aide à la décision important en fournissant des statistiques et documents cartographiques qui permettent la prise en compte et l'appréciation de la valeur du patrimoine naturel dans la gestion raisonnée d'un territoire. L'évaluation qualitative que représente l'inventaire ZNIEFF peut ainsi aider à une meilleure cohérence des opérations d'aménagement et de mise en valeur du territoire.

Sans avoir de caractère juridique, les ZNIEFF sont progressivement intégrées dans les problématiques d'aménagement du territoire et sont de plus en plus prises en compte 
dans les Plans d'Occupation des Sols, études d'impact, schémas directeurs, plans d'aménagement préalables aux décisions d'aménagement ou de protection tant sur le plan régional que national.

Les ZNIEFF constituent une base de réflexion et de travail essentielle afin de mener des politiques spécifiques à certains milieux (zones humides, pelouses sèches...), des synthèses thématiques pouvant être réalisées. Elles constituent à cet égard une base essentielle pour la délimitation de zones particulières dans le cadre de la constitution d'un réseau national d'espaces protégés, de l'application des directives européennes en matière de protection des espèces et des milieux (directive Habitat, directive Oiseaux, " article 19 »...) ou de conventions internationales ratifiées par la France (convention de Ramsar...).

Elles représentent en outre un outil d'observation scientifique très important pour le suivi d'indicateurs biologiques propres à rendre compte des évolutions des écosystèmes.

\section{Les objectifs des parcs naturels régionaux}

Le concept de parc naturel régional est né à la DATAR en 1965 dans le contexte d'une extension rapide des grandes agglomérations urbaines et d'un exode rural accéléré. Conçu à la fois comme une sorte de " poumon vert » pour les citadins et comme un moyen de revitaliser des espaces ruraux menacés, le Parc naturel doit alors présenter " un intérêt particulier, par la qualité de son patrimoine naturel et culturel, pour la détente, le repos des hommes et le tourisme ». Ce concept prendra corps le 1er mars 1967 avec la parution du décret instituant les Parcs naturels régionaux.

Tour à tour les différents épisodes de la vie politique française - régionalisation puis décentralisation -, l'évolution des conditions socio-économiques du développement régional - déprise agricole, dévitalisation de certains espaces ruraux - et l'apparition au premier plan de nouvelles préoccupations - telles que la protection de l'environnement et plus récemment la mise en valeur du patrimoine et des paysages -, infléchiront les vocations des Parcs naturels dans de nouvelles directions. Le décret de 1988, pris en application de la loi dite de « décentralisation », précisera leurs missions :

- protéger le patrimoine naturel et culturel ;

- contribuer au développement économique et social ;

- promouvoir l'accueil, l'éducation et l'information du public ;

- réaliser des actions expérimentales ou exemplaires dans ces domaines et contribuer à des programmes de recherche.

Il ne s'agit plus dès lors de satisfaire des besoins extérieurs mais d'énoncer un projet de gestion globale d'un territoire qui mette en cohésion l'ensemble de ces objectifs, permettant ainsi de concilier protection de l'environnement, mise en valeur du patrimoine et développement régional - mission parfois mal comprise ou mal acceptée de la part de nombre d'élus locaux qui perçoivent souvent les opérations de protection de la nature et du patrimoine comme un frein au développement local. Les Parcs deviennent ainsi des instruments essentiels des politiques de protection de l'environnement et d'aménagement du territoire, vocation récemment réaffirmée par la loi du 8 janvier 1993 sur la protection et la mise en valeur des paysages, où ils 
constituent « un cadre privilégié des actions menées par les collectivités publiques en faveur de la préservation des paysages et du patrimoine naturel et culturel ».

Aujourd'hui, l'Institution-Parc compte 26 Parcs naturels régionaux répartis sur 20 régions, 49 départements et 2063 communes. Ils couvrent plus de $40000 \mathrm{~km} 2$ soit $8 \%$ du territoire national métropolitain, et présentent un échantillonnage varié des milieux naturels et paysages français : forêts périurbaines, de moyenne ou haute montagne (Haute vallée de Chevreuse, Forêt d'Orient, Landes de Gascogne, Morvan), bocage (Normandie-Maine), zones humides (Brière, Camargue, Marais Poitevin), moyenne ou haute montagne (Vosges du Nord, Ballons des Vosges, Haut-Jura, Queyras), littoral (Armorique, Corse), ou d'autres plus spécifiques comme les Volcans d'Auvergne.

Les objectifs contenus dans leurs Chartes sont multiples et traduisent bien la dualité de cette politique, entre protection de l'environnement et développement régional. Sauvegarde et réhabilitation du patrimoine vernaculaire, remise en valeur et promotion des activités traditionnelles, savoir-faire et produits régionaux, animation culturelle et pédagogique, préservation de milieux naturels, paysages remarquables et représentatifs constituent la plus grande part des actions menées dans les Parcs naturels.

Parmi la vingtaine de projets actuellement à l'étude, la majorité concernent des espaces au caractère rural très marqué où les objectifs de revitalisation économique et de mise en valeur du patrimoine sont affirmés. La fonction de coupure verte, sorte de « zone de contention » de l'extension urbaine est cependant toujours à l'oeuvre. En effet, le quart des projets s'appliquent à des espaces périphériques de grandes agglomérations : le Grand Bassin parisien bien sûr (Boucles de la Marne et de l'Ourcq, Gâtinais, Picardie, Perche, Vexin français), la conurbation lilloise (extensions du Parc Nord-Pas-de-Calais), mais aussi la vallée de la Loire (Val de Loire-Anjou-Touraine).

Notons enfin l'apparition de projets en Région Centre jusqu'ici absente de ce dispositif, apportant ainsi un rééquilibrage territorial de l'Institution-Parc, mais aussi l'absence inquiétante de projets dans les départements méditerranéens toujours soumis à une pression urbaine et touristique extrêmement forte et non maîtrisée.

\section{NOTES}

1. Le ministère de la Protection de la Nature et de l'Environnement est créé le 2 février 1971. Robert Poujade est nommé ministre délégué auprès du Premier ministre.

2. L'administration centrale ne compte que 580 agents. Les services décentralisés sont représentés par les DIREN qui regroupent un nombre limité d'agents (de 15 à 50 suivant les régions).

3. Ces préoccupations se retrouvent dans le nouvel organigramme du ministère qui distingue trois Directions thématiques : la Direction de l'Eau, la Direction de la Nature et des Paysages, la Direction de la Prévention des Pollution et des Risques.

4. A titre d'illustration de cette amplitude du champ de l'environnement, la loi sur la protection de la nature de 1976 a nécessité la signature de onze ministres aux compétences fort différentes : Intérieur, Justice, Défense, Equipement, Agriculture, Santé, Qualité de la vie, Industrie et Recherche, Transports, Culture, DOM-TOM.

5. L'État de l'Environnement, rapport d'activité du ministère édité depuis 1979, fournit à cet égard une bonne synthèse de l'étendue de ses domaines de compétences. 
6. Ce besoin s'est récemment affirmé avec force dans le cadre de la redéfinition de la politique des Parcs naturels régionaux. Mais une telle demande ne s'exprime pas seulement dans le domaine de l'environnement ; le même type de besoin émane également des domaines de l'aménagement du territoire, de la culture et du patrimoine, favorisé ou suscité par l'insertion des politiques nationales dans le concert international, européen en particulier, où les diverses dotations et subventions sont attribuées régionalement, exigeant de la part des Etats-membres des sélections a priori de territoires éligibles.

7. C'est-à-dire la désignation et la hiérarchisation des zones et types d'espaces dans lesquels va être appliquée telle ou telle politique.

8. Recensement général de la population, Recensement général de l'agriculture, TERUTI, Inventaire Communal, etc.

9. Pour faire un parallèle avec le côté culturel du patrimoine national, rappelons que depuis 1965, sous l'impulsion du ministre de la Culture André Malraux, a débuté l'Inventaire général du patrimoine et des richesses artistiques de la France. Sa mission principale est de « recenser, d'étudier et de faire connaître toute oeuvre qui, du point de vue artistique, historique, archéologique ou ethnologique constitue le patrimoine national ».

10. En 1978, Raymond Barre, alors Premier ministre, institue la Commission interministérielle des comptes du Patrimoine naturel en vue de la « mise en oeuvre progressive mais complète d'un système de comptes du patrimoine naturel ». Cette idée sera réaffirmée avec force dans les conclusions d'un rapport au ministre (Huguette Bouchardeau) remis en 1983 par Edgard Pisani et intitulé Pour la définition, la protection et la gestion d'un réseau d'espaces naturels. Il préconisait d'établir « les inventaires indispensables à la connaissance des richesses naturelles ». Plus tard, Brice Lalonde, alors ministre de l'Environnement lancera l'idée d'un Observatoire du patrimoine naturel. Ce dernier objectif est en cours de réalisation avec la création en 1992 de l'Institut Français de l'Environnement.

11. Comme la Convention de Ramsar relative aux zones humides d'importance internationale.

12. Le Secrétariat de la Faune et de la Flore est créé en 1979 au sein du Muséum national d'Histoire naturelle. Il a pour mission d'organiser et de développer la constitution d'une collection de données scientifiques ou bibliographiques sur la faune et la flore.

13. La Directive CEE 79/409 relative à la protection des oiseaux sauvages prévoit la désignation des ZICO : Zones d'Importance Communautaire pour les Oiseaux. La Directive CEE 92/43 « Habitat, faune, flore » prévoit la désignation de zones naturelles à maintenir devant s'insérer dans un réseau écologique européen appelé « Réseau Natura $2000 »$.

14. Le décret portant procédure d'inventaire du degré de pollution des eaux superficielles ne paraîtra en effet qu'en 1969.

15. Sans que l'on puisse toutefois leur accorder une validité universelle. C'est ici l'un des débats de fond des problèmes d'environnement auquel le monde scientifique, $\mathrm{y}$ compris dans le domaine de la santé, ne peut dans l'état actuel des connaissances apporter de certitudes absolues.

16. Voir Paoli Dominique et Rieu Thierry, «La situation de l'eau en France », Économie et Statistique , 258-259, 1992, pp. 95-104. 
17. Ainsi, à la demande du ministère de l'Environnement en 1974, le SCEES, service statistique du ministère de l'Agriculture, a complété son enquête annuelle sur la répartition du territoire agricole, qui existait depuis 1965, par des données sur l'occupation du territoire à des fins non agricoles (voir enquête TERUTI). En 1979, c'est dans l'Inventaire communal produit par le SCEES et l'INSEE que de nouvelles rubriques seront introduites.

18. L'État de l'Environnement, édité annuellement par le ministère pour dresser un état général de la situation de l'environnement en France et un bilan de ses actions illustre parfaitement cette dualité. Il est, de fait, très largement alimenté de données issues d'autres ministères (Agriculture, Mer, Industrie et Commerce, Equipement, Tourisme, Santé...) et organismes nationaux (SCEES, INSEE, CEA, SNCF, EDF...) ou internationaux (EUROSTAT, OCDE).

19. Jollivet Marcel et Pavé Alain (1993).

20. Ainsi, l'ancienne Mission du Paysage, cellule autonome dans l'ancien organigramme du ministère, qui remplissait un service de mission et de promotion d'actions sur le paysage, est intégrée dans le nouvel organigramme au sein de la Direction de la Nature et des Paysages et transformée en Bureau du Paysage dont les actions sont désormais davantage tournées vers la gestion et l'application de mesures prises dans le cadre de la loi du 8 janvier 1993 sur la protection et la mise en valeur des paysages. Par ailleurs, il faut souligner, là aussi, les problèmes de répartition des compétences qui existent avec d'autres ministères, en particulier ceux de l'Équipement et de la Culture, qui ont également leur propre politique en matière de paysage et de patrimoine. Aussi le projet actuel de regrouper tout ce qui a trait aux sites et paysages sous la tutelle de l'Environnement, et tout ce qui a trait au patrimoine architectural, monumental et vernaculaire sous la tutelle de la Culture va dans le sens d'une redéfinition et d'une nouvelle délimitation des compétences et missions de chacun.

21. Décret du 25 avril 1988 relatif aux parcs naturels régionaux et décret du 1er septembre 1994 pris pour l'application de la loi du 8 janvier 1993 sur la protection et la mise en valeur des paysages.

22. Une vingtaine de projets sont en cours d'examen.

23. Comme ce fut le cas pour les réserves naturelles avec « l'objectif 100 Réserves naturelles ».

24. Créé en 1992, l'IFEN est chargé de fonctions de surveillance et d'évaluation de l'environnement. Ses missions sont précisément définies : " procéder à des enquêtes, à la définition et au suivi d'indicateurs, à la gestion de données statistiques, au développement de modèles de prévision ou d'évaluations économiques, à l'animation d'observatoire ». Depuis 1990, plusieurs groupes de travail ont été mis en place à cet effet : projet de base de données BRIDGE, puis projet IARE. Le laboratoire STRATES, associé à la SEGESA a été également sollicité dans le cadre d'une réflexion sur l'élaboration d'une critériologie du paysage : cf. Bontron Jean-Claude, Brochot Aline et Luginbühl Yves (1994).

25. Cf. Guide du diagnostic pour le développement rural, 1991.

26. Cf. Rapport sur l'identification des zones rurales fragiles en France, Paris, Segesa/Datar, 1987 et Essai de typologie socio-économique des cantons français, Segesa/Datar, Paris, mars 1993.

27. Programme européen d'évaluation de l'occupation des terres à partir d'images de la télédétection. Les satellites permettent l'observation fine du territoire avec une résolution spatiale de 20 mètres pour SPOT et 30 mètres pour LANDSAT et la 
répétitivité des observations pour un même espace (tous les 1 à 4 jours pour SPOT et tous les 16 jours pour LANDSAT). La portion de territoire couverte à chaque prise de vue varie également de $60 \mathrm{~km} \mathrm{X} 60 \mathrm{~km}$ pour SPOT à $185 \mathrm{~km} \mathrm{X} 185 \mathrm{~km}$ pour LANDSAT. Les progrès technologiques se succédant à des rythmes rapides, de nouvelles générations de satellites d'observation sont déjà à l'oeuvre comme ERS1, satellite européen de télédétection, et ERS2 à partir de 1995. L'Agence spatiale européenne quant à elle annonce le lancement en 1998 d'un satellite de surveillance de l'environnement baptisé ENVISAT.

28. Larrue Corinne \& Knoepfel Peter (1993).

29. Bontron Jean-Claude, Brochot Aline \& Luginbühl Yves, op. cit.

\section{RÉSUMÉS}

Depuis une vingtaine d'années, le champ de la politique de l'environnement s'est considérablement élargi. Chaque segment de cette politique s'est appuyé sur la construction d'outils de connaissance et d'observation spécifiques qui composent aujourd'hui un système d'information complexe et varié, mais qui ne suffit pas à une appréciation globale des phénomènes d'environnement sur tout le continuum territorial. Aussi de nouveaux modes d'appréhension et d'évaluation doivent-ils être recherchés qui permettent, par la mise en cohérence de l'ensemble des données disponibles sur la totalité du territoire, une vision globale et nationale de la qualité environnementale des espaces français.

Territorial designs of environmental policies in France. Which tools for evaluation and decisionmaking?

During the last twenty years, French environmental policies have greatly widened their field of intervention. Each step of the process has required the sharpening of adequate tools intented to gather specific observations and knowledges. As a result, a complex and diverse information system has been set up. But, nowadays, this information system appears to be insufficient to draw up a global appraisal of the environmental contexts covering the whole territorial continuum. New ways of evaluation and assessment need to be improved to consider consistently all the data available over the whole territory and to be able to get a global and national vision of the environmental quality of French spaces.

Territorializar la política del medio ambiente en Francia. ¿Cuáles son las herramientas para la evaluación y la ayuda a la decisión?

Desde hace unos veinte años, el sector de la política del medio ambiente se ha extendido considerablemente. Cada segmento de esta política se ha fundado en la construcción de herramientas de conocimiento y observación específicas que componen actualmente un sistema de información complejo y variado, pero que no son suficientes para construir una estimación global de los fenómenos del medio ambiente en todo el continuum territorial. Habría que buscar además nuevos mecanimos de aprehensión y evaluación que permitan, mediante un desarrollo que dé coherencia al conjunto de datos disponibles en la totalidad del territorio, una visión global y nacional de la calidad medio ambiental de los espacios franceses. 
INDEX

Mots-clés : Environnement, Politique, Territorialisation, Outils, Méthodes, Données, Evaluation Keywords : France, environment, Policy, «Territorialization », Tools, Methods, Data

Palabras claves : medio ambiente, Francia, Política, Territorialisación, Herramientas, Métodos, Datos, Evaluación

\section{AUTEUR}

\section{ALINE BROCHOT}

Géographe, ingénieur au CNRS (STRATES). Elle travaille sur la qualification des espaces français en termes de valeur environnementale, patrimoniale, paysagère. Elle a notamment contribué au rapport Les Indicateurs du paysage. Etude de cadrage, faisabilité d'une critériologie, remis à l'Institut français de l'environnement en 1994. 